\title{
Cationic Carbon Nanotubes Bind to CpG Oligodeoxynucleotides and Enhance Their Immunostimulatory Properties
}

Alberto Bianco, ${ }^{*}{ }^{\dagger}$ Johan Hoebeke, ${ }^{\dagger}$ Sylvie Godefroy, ${ }^{\dagger}$ Olivier Chaloin, ${ }^{\dagger}$ Davide Pantarotto, ${ }^{\dagger, \S}$ Jean-

Paul Briand, ${ }^{\dagger}$ Silviane Muller, ${ }^{\dagger}$ Maurizio Prato, ${ }^{*, \S}$ and Charalambos D. Partidos ${ }^{*}, \dagger$

${ }^{\dagger}$ Institut de Biologie Moléculaire et Cellulaire UPR9021 CNRS, Immunologie et Chimie Thérapeutiques, 67084, Strasbourg, France

Email : $\underline{\text { H.Partidos@ibmc.u-strasbg.fr; }}$ A.Bianco@ibmc.u-strasbg.fr

${ }^{\S}$ Dipartimento di Scienze Farmaceutiche, Università di Trieste, 34127 Trieste, Italy

Supporting Information 


\section{Experimental Section}

Synthesis of $\boldsymbol{f}$-CNT. HiPCO, single-walled carbon nanotubes (CNT Lot \# HPR-86) were purchased from Carbon Nanotechnologies, Inc. (USA). Cationic $f$-CNT 1 and $f$-CNT 2 were prepared as described in the literature. ${ }^{1,2}$

\section{Surface plasmon resonance}

The BIAcore 3000 system, sensor chip CM5, surfactant P20, amine coupling kit containing Nhydroxysuccinimide (NHS) and N-ethyl-N'-dimethylaminopropyl carbodiimide (EDC) were from BIAcore (Uppsala, Sweden). All biosensor assays were performed in HBS running buffer (20 mM HEPES, $20 \mathrm{mM}$ sodium acetate, $140 \mathrm{mM}$ potassium acetate, $3 \mathrm{mM}$ magnesium acetate, $0.02 \%$ surfactant P20, $\mathrm{pH}$ 7.3). Immobilization of nanotubes was performed by injecting $35 \mu \mathrm{l}$ of CNT (100 $\mu \mathrm{g} / \mathrm{ml}$ in acetate buffer, $\mathrm{pH} 4.0)$ onto the surface of a sensor chip CM5 activated with EDC/NHS. This was followed by $20 \mu \mathrm{l}$ of ethanolamine hydrochloride, $\mathrm{pH} 8.5$, to saturate the free active sites of the matrix. A $100 \mathrm{mM} \mathrm{H}_{3} \mathrm{PO}_{4}$ solution was used to remove CNT non-covalently immobilized on the chip. All the binding experiments were carried out at $25^{\circ} \mathrm{C}$ with a constant flow rate of $30 \mu \mathrm{l} / \mathrm{min}$. ODN CpG at concentrations from $10 \mathrm{mg} / \mathrm{mL}$ to $0.625 \mathrm{mg} / \mathrm{mL}$ were fluxed over the immobilized nanotubes for 90 seconds and their disssociation was followed in the HBS buffer for 240 seconds. The immobilized CNT were regenerated for 30 seconds with $3 \mathrm{M} \mathrm{MgCl}_{2}$. The kinetic parameters were calculated using the BIAeval 3.1 software. Analysis was performed using the simple Langmuir binding model. The specific binding profiles were obtained after subtracting the response signal from the channel control and subtracting the response of the solvent (Figure 1). The fitting of the model was judged by the chi square value and randomness of residue distribution compared to the theoretical model. 


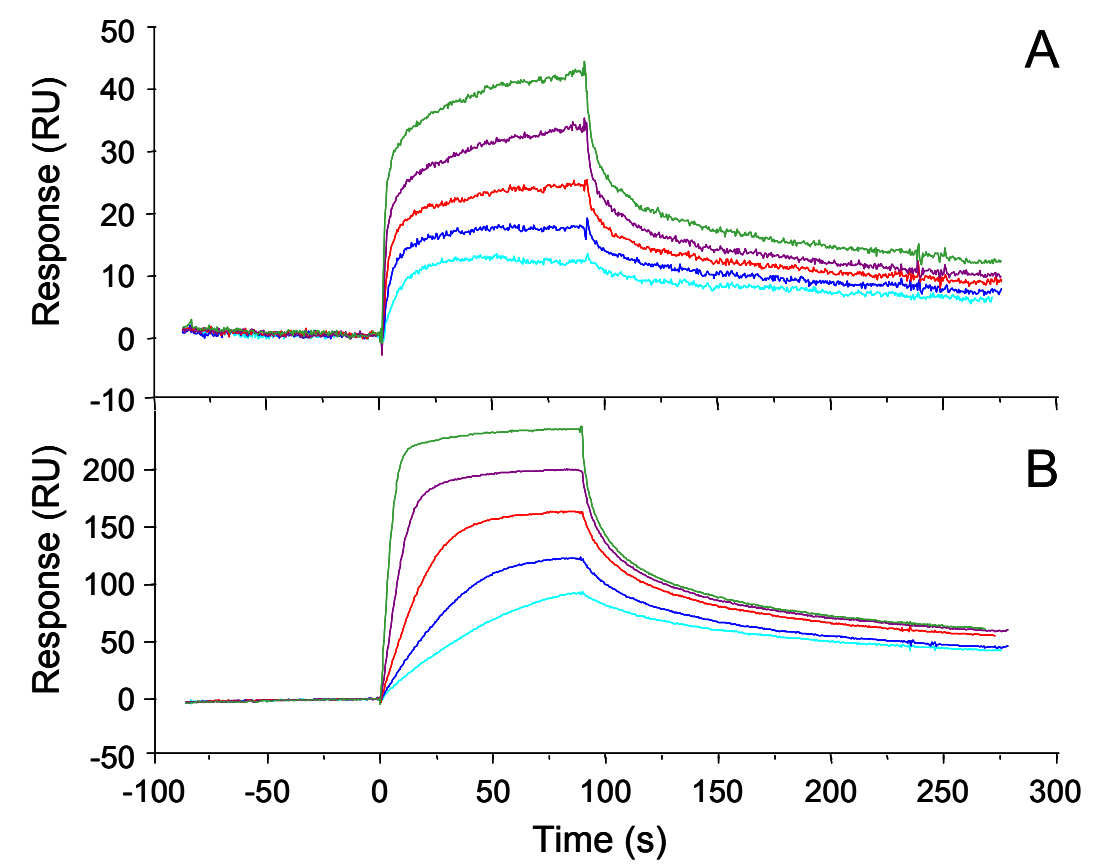

Figure S1. Sensorgrams obtained by allowing various concentrations of analyte (ODN CpG 1668) to react with the $f$ CNT 1 (A) or $f$-CNT 2 (B) immobilized onto sensor chip. RU corresponds to the resonance unit $\left(1000 \mathrm{RU}=1 \mathrm{ng} / \mathrm{mm}^{2}\right.$ of analyte) [10 nM (cyan), $20 \mathrm{nM}$ (blue) $41 \mathrm{nM}$ (red) $82 \mathrm{nM}$ (magenta) $163 \mathrm{nM}$ (green)].

\section{Lymphocyte stimulation assay}

Spleens from naïve mice were aseptically removed and a single cell suspension was prepared in RPMI-1640 medium (Life Technologies, Cergy-Pontoise, France) supplemented with $100 \mathrm{IU} / \mathrm{ml}$ gentamycin, $25 \mathrm{mM}$ HEPES, and 1\% heat-inactivated autologous mouse serum. 4 x 105 viable splenocytes were cultured in the presence of various concentrations of CNT alone or together with a constant concentration of ConA $(0.5 \mathrm{mg} /$ culture $)$ in $5 \% \mathrm{CO}_{2}$ at $37^{\circ} \mathrm{C}$ for three days. In experiments conducted to study the immunopotentiating effect of CNT, a constant concentration

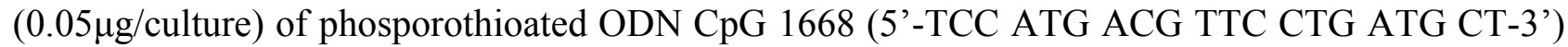
or ODN CpG 1982 (5'-TCC AGG ACT TCT CTC AGG TT-3'), which is non-immunostimulatory in vitro ${ }^{3}$ and was used as control (purchased from MWG Biotech, Munich, Germany), were preincubated with various concentrations of each of the tested CNT for 30min at room temperature (giving an excess of CNT over the ODN CpG of 18:1, 9:1, 4.5:1, 2.25:1 and 1.12:1) before addition of the complexes into the tissue culture. At 18:1 ratio, the concentration of CNT was $5 \mu \mathrm{g} / \mathrm{culture}$. 
Eighteen hours before the end of the culture, cells were pulsed with $1 \mathrm{mCi}$ of ${ }^{3}[\mathrm{H}]$-thymidine $(6.7 \mathrm{mCi} / \mathrm{mmol}, \mathrm{ICN}$, Orsay, France) and incorporation was measured using a Matrix 9600 direct beta counter (Packard, Downers Grove, IL). Results were expressed as mean counts per minute (c.p.m) from triplicate cultures \pm standard deviation (sd) or as percentage (\%) increase of the immunostimulatory properties of ODN CpG. The latter was calculated as follows; (c.p.m in the presence of CNT/ODN CpG complexes - c.p.m with medium alone) / (c.p.m in the presence of ODN CpG - c.p.m with medium alone) x 100 .

\section{Cytokine secretion}

Culture supernatants collected after three days incubation were assayed for the presence of IFN- $\gamma$ or IL-6 using a double-sandwich ELISA and commercial antibodies from PharMingen and polyvinyl Falcon plates. All steps were performed according to manufacturer recommendations. Results were expressed as mean cytokine concentration $(\mathrm{pg} / \mathrm{ml}) \pm$ standard deviation $(\mathrm{SD})$ after extrapolation from a standard curve prepared with a reference cytokine (PharMingen) for the antigen concentration tested in triplicates. In tests were IL-6 secretion was measured after stimulation of naïve lymphocytes with CNT/ODN CpG complexes, supernatants were assayed at 1/3 and 1/10 dilution and the \% decrease of IL-6 secretion was measured with reference to IL-6 production stimulated by the ODN CpG 1668 alone. The values presented in the text are average \% decrease obtained after screening the supernatants at $1 / 3$ and $1 / 10$.

\section{References}

1. Georgakilas, V.; Tagmatarchis, N.; Pantarotto, D.; Bianco, A.; Briand, J.-P.; Prato, M. Chem. Commun. 2002, 3050-3051.

2. Pantarotto, D.; Partidos, C. D.; Hoebeke, J.; Brown, F.; Kramer, E.; Briand, J.-P.; Muller, S.; Prato, M.; Bianco, A. Chem. Biol. 2003, 10, 961-966.

3. Davis, H. L.; Weeratna, R.; Waldschmidt, T. J.; Tygrett, L.; Schorr, J.; Krieg, A. M.; Weeranta, R. J. Immunol. 1998, 160, 870-876. 